\title{
PyNeb: a new software for the analysis of emission lines
}

\author{
Valentina Luridiana $^{1,2}$, Christophe Morisset ${ }^{1,3}$ and Richard A. Shaw ${ }^{4}$ \\ ${ }^{1}$ Instituto de Astrofísica de Canarias, \\ Vía Láctea s/n, La Laguna, E-38205, Spain \\ ${ }^{2}$ Departamento de Astrofísica, Universidad de La Laguna, \\ La Laguna, E-38205, Spain \\ ${ }^{3}$ Instituto de Astronomía, UNAM, \\ Apartado Postal 70 - 264, México, D.F., 04510, Mexico \\ ${ }^{4} \mathrm{NOAO}$, Tucson, AZ 85719, USA \\ email: vale@iac.es; chris.morisset@gmail.com; shaw@noao.edu
}

\begin{abstract}
We describe an ongoing project to migrate the emission-line analysis package nebular from its current environment, IRAF, to a modern programming environment where it can be used from command line, a local GUI, or the web. We are also updating the supporting atomic data where they have been superseded by superior calculations or measurements.
\end{abstract}

Keywords. Atomic data, ISM: abundances, methods: data analysis

\section{Introduction}

The nebular package is an IRAF tool for the analysis of collisionally excited emission lines (Shaw \& Dufour 1995). It enjoys wide use in the research community for the analysis of physical conditions and chemical abundances in a variety of astrophysical contexts, including active galaxies, H II regions, and PNe. It is also widely used by people who model gaseous nebulae with photoionization codes as a starting point.

In nebular, an atom is represented as an $n$-level system. As described by Shaw et al. (1998), the software is data-driven, so that the input atomic data are not hardwired and can easily be changed without re-compiling the source code: the energy levels, statistical weights, transition probabilities, and collision strengths are all read from FITS files at run-time, allowing easy comparisons among different data sources. Also, for a given ion, users may employ any recognized line ratio by creating an expression for the desired transitions. There is also a choice of interstellar extinction laws to deredden data.

Recently, we embarked on a project to recast the nebular source code base to a modern programming environment, python, in order to enhance portability, to allow the implementation of GUI and web interfaces, and to make it simple for developers or advanced users to extend the original functionality of the code. The successor of nebular is called PyNeb and will provide the same functionality of nebular with a user-friendly interface, a vast range of visualization tools, and full access to the intermediate quantities of the calculation. New analysis features will also be added.

\section{Current state of PyNeb}

PyNeb is still under development, but it already features many functionalities and visualization tools, including most original nebular functionalities. As an example, we show a plot of emission-line diagnostics obtained from the spectrum of a single object 


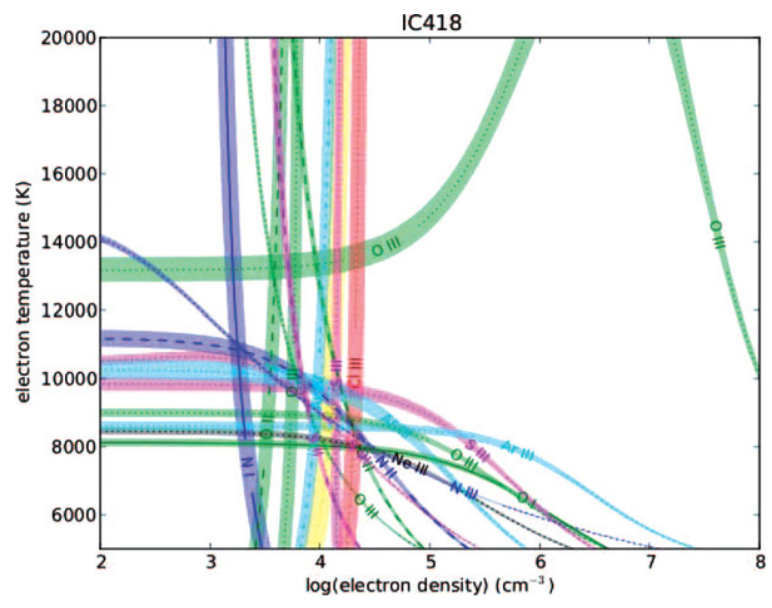

Figure 1. Emission-line diagnostic plot of a planetary nebula.

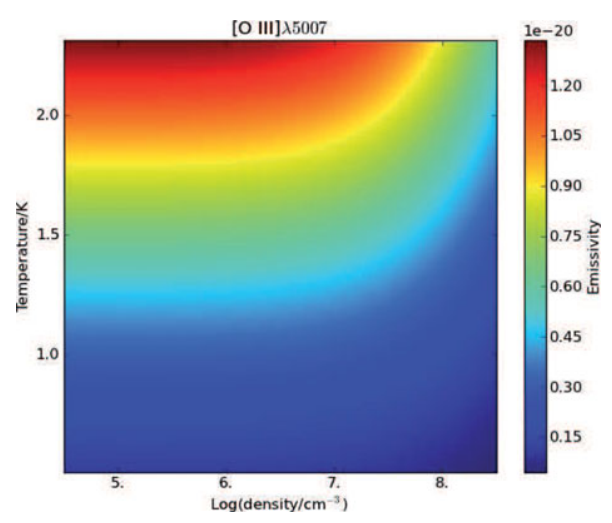

Figure 2. Emissivity map of $[\mathrm{O}$ III $] \lambda 5007$ as a function of physical conditions.

(Fig. 1) and the emissivity map of [O III] $\lambda 5007$ as a function of temperature $\left(T_{e}\right)$ and density $\left(N_{e}\right)$ (Fig. 2). Among other features, the code can also generate contour maps of diagnostic line ratios as a function of $T_{e}$ and $N_{e}$; perform simultaneous determinations of $T_{e}$ and $N_{e}$ from pairs of line ratios; and easily switch among different atomic data.

\section{Future developments}

Some of the intended enhancements include: add new ions, including $s$-process elements; determine abundances for $\mathrm{He}$ and other light ions from recombination lines; perform the error analysis of $T_{e}, N_{e}$ and abundances; add recipes for computing total elemental abundances from ionic abundances, using common or user-defined ICF formulae; and create VO-compatible web services to provide PyNeb over the internet.

\section{References}

Shaw, R. A., et al. 1998, in R. Albrecht et al., (Eds.), ADASS VII ASP Conf. Ser., 192, 145

Shaw, R. A. \& Dufour, R. J. 1995, PASP, 107, 896 